\title{
New model linking periodontitis and RA
}

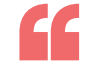

...Aa induces activation of citrullinating enzymes in neutrophils
The periodontal pathogen Aggregatibacter actinomycetemcomitans $(A a)$ has been identified as a candidate bacterial trigger for rheumatoid arthritis (RA), providing a link between periodontal infection and autoimmunity. The new study published in Science Translational Medicine proposes a model in which $A a$ induces activation of citrullinating enzymes in neutrophils and thus autoantigen production.

"While protein citrullination and periodontal disease appear to be relevant for RA pathogenesis,

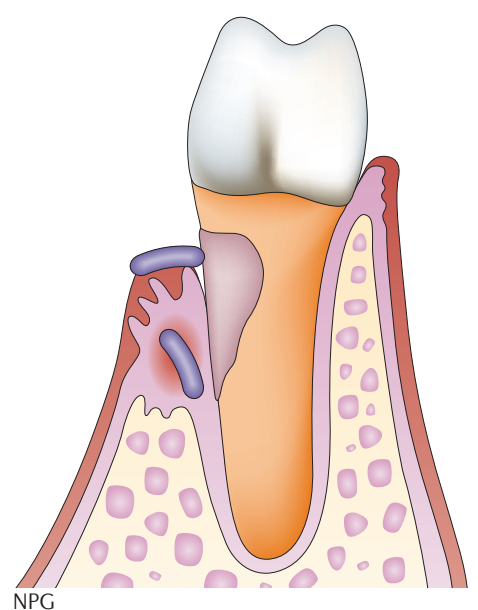

our understanding of how these processes may be mechanistically related remained poorly defined", says corresponding author Felipe Andrade. Using mass spectrometry analysis, Andrade's group found that the patterns of hypercitrullination in gingival crevicular fluid from patients with periodontal disease mirrored those seen in synovial fluid from patients with RA. Analysis of these citrullination patterns suggested they were probably driven by endogenous peptidylarginine deiminases (PADs) within host immune cells, and not by bacterial citrullinating enzymes. "This observation dramatically shifted our approach to identifying relevant bacterial species with the capacity to activate PAD enzymes in human cells," explains co-author Maximilian Konig.

Among the different periodontal pathogens and oral commensals investigated, only $A a$ could induce hypercitrullination of human neutrophils. "We show that $A a$ induces neutrophil hypercitrullination through the secretion of leukotoxin A (LtxA), a bacterial pore-forming toxin that induces calcium influx and subsequent hyperactivation of PAD enzymes in the neutrophil," remarks Andrade.

In the clinic, $47 \%$ of patients with RA showed evidence of previous Aa infection, compared with $11 \%$ of controls; furthermore, anti-LtxA antibodies were associated with both rheumatoid factor (RF) and anticitrullinated protein antibody (ACPA) positivity in patients with RA. HLA-DRB1 shared epitope alleles the strongest known genetic risk factor for RA - were associated with RF and ACPA only in those patients showing evidence of previous or current $A a$ infection. "It appears as if you need both the right genetic background to present citrullinated proteins and an infection that drives the production of citrullinated autoantigens, such as $A a$, to develop disease-specific antibodies and possibly RA," reasons Konig. "Clinical studies will be critical to guide our understanding on how to translate these findings to the bedside," he concludes.

Jessica McHugh

ORIGINAL ARTICLE Konig, M. F. et al. Aggregatibacter actinomycetemcomitans-induced hypercitrullination links periodontal infection to autoimmunity in rheumatoid arthritis. Sci. Transl. Med. http://dx.doi.org/ 10.1126/scitranslmed. aaj1921 (2016) 